\title{
Including Quantum Effects in the Dynamics of Complex (i.e., Large) Molecular Systems
}

\author{
William H. Miller
}

\author{
Department of Chemistry and K. S. Pitzer Center for Theoretical Chemistry, University of \\ California, and Chemical Sciences Division, Lawrence Berkeley National Laboratory \\ Berkeley, California 94720-1460
}

\begin{abstract}
The development in the 1950's and 60's of crossed molecular beam methods for studying chemical reactions at the single-collision molecular level stimulated the need and desire for theoretical methods to describe these and other dynamical processes in molecular systems. Chemical dynamics theory has made great strides in the ensuing decades, so that methods are now available for treating the quantum dynamics of small molecular systems essentially completely. For the large molecular systems that are of so much interest nowadays (e.g. chemical reactions in solution, in clusters, in nano-structures, in biological systems, etc.), however, the only generally available theoretical approach is classical molecular dynamics (MD) simulations. Much effort is currently being devoted to the development of approaches for describing the quantum dynamics of these complex systems. This paper reviews some of these approaches, especially the use of semiclassical approximations for adding quantum effects to classical MD simulations, also showing some new versions that should make these semiclassical approaches even more practical and accurate.
\end{abstract}

\section{Introduction.}

The introduction of crossed molecular beam methods into chemistry ${ }^{1}$ in the 1950's and 60's - by Taylor and Datz, ${ }^{2}$ Green and Ross et al. ${ }^{3}$ Herschbach et al. ${ }^{4}$ Bernstein et al..${ }^{5}$ and finally in full flower by Y.T. Lee ${ }^{6}-$ revolutionized the way chemists think about chemical reactions. One began to study and think about chemical kinetics and dynamics at the molecular level, very much in the same spirit with which physical chemists had used molecular spectroscopy for many years to study molecular structure. These new developments in experimental capabilities thus spurred many young theoretically inclined persons of my generation to take up the challenge of developing the theoretical models for understanding these new experiments qualitatively, and the theoretical methods for carrying out calculations to describe them quantitatively. The first efforts in this regard were largely translating the quantum scattering methodology developed years earlier in nuclear physics into the arena of molecular problems, but before long new theoretical approaches were developed specifically in the area of chemical dynamics (some of which even made the reverse trip back into physics applications.)

Theoretical chemical dynamics has made enormous strides in the last 40 years. Fully rigorous quantum mechanical calculations ${ }^{7}$ are now almost routine for the simplest chemical reactions, atom + diatom $(\mathrm{A}+\mathrm{BC})$, and are increasingly possible for somewhat larger molecular systems. These calculations are limited in accuracy only by that of the underlying Born- 
Oppenheimer potential energy surface (PES). These rigorous quantum calculations are important not only for making definitive comparisons with experimental results, but they also provide the benchmarks results that are necessary to validate more approximate theoretical approaches that can be applied to even more complex molecular systems.

Classical trajectory simulation methods - which were introduced earlier by Alder et al. ${ }^{8}$ to describe statistical mechanical systems - were first applied ${ }^{9}$ to the simplest chemical reactions ('A + BC') in the 1960's, and today these classical molecular dynamics (MD) methods are probably the most widely used theoretical tool of all. They are applied to treat chemical reactions in solution, in clusters, in proteins (and other biomolecular systems), in solids, etc. In talking with Alder ${ }^{10}$ recently, he mentioned that billion atom MD simulations are now possible!

Semiclassical (SC) theory also had its beginnings in the late 1950's and '60's, with the work of Ford and Wheeler ${ }^{11}$ showing how quantum effects in elastic scattering can by described by use of the WKB approximation to the Schrödinger equation; a very comprehensive review by Bernstein ${ }^{12}$ showed how all quantum effects in elastic scattering can be described within a SC framework. The earliest work of Lee ${ }^{13}$ in his first independent faculty position, at the University of Chicago, was in fact a very comprehensive studies of elastic scattering differential cross sections of rare gas atoms amongst themselves, displaying the full array of quantum effects: rainbows, supernumerary rainbows, diffraction oscillations, and identical particle interference structure. These interference features were very important in determining quantitative pair potentials for the rare gas atoms from the experimental measurements.

In the 1970's it was shown how semiclassical theory could be generalized to describe inelastic and reactive scattering. ${ }^{14,15}$ (The challenge here was to learn how numerically computed classical trajectories could be used as input into a multi-dimensional generalization of the WKB approximation.) Since this description includes interference and tunneling (or classically forbidden processes in general, which arise as the analytic continuation of interference), all quantum effects in molecular dynamics are incorporated in the SC description at least qualitatively, and usually quite quantitatively. E.g., quantization of bounded motion and selection rules due to symmetry of identical particles arise as interference effects in SC theory.

Much of chemistry, and molecular science in general is focused nowadays on large molecular systems, such as chemical reactions in complex environments as noted above, and the most generally available approach for describing/modeling them is classical molecular dynamics. There are situations, however, for which quantum effects will be significant even in these complex systems. For example, consider the water molecule, which is ubiquitous in bio simulations: the zero-point energy in its two $\mathrm{OH}$ stretching vibrations and its bending vibration is more than 20 times $\mathrm{k}_{\mathrm{B}} \mathrm{T}$ (at $300 \mathrm{~K}$ ). If this energy were allowed to behave classically in a simulation with hundreds (or thousands) of water molecules, it is clear that nonsense would result; the energy could leak out of these modes classically (which it cannot do quantum mechanically) and boil the system!

Of course, most persons who carry out classical MD simulations are well aware of this, and water is usually treated as a rigid molecule that can rotate and thus realign its dipole moment and solvate a reaction coordinate appropriately, for example, but is not allowed to vibrate. But often 
in an $\mathrm{H}$ atom transfer reaction - for example, in a biomolecular system, $\mathrm{AH}+\mathrm{B} \rightarrow \mathrm{A}+\mathrm{HB}$, where an $\mathrm{H}$ atom moves from site $\mathrm{A}$ to site $\mathrm{B}$ - it is not the $\mathrm{H}$ atom on $\mathrm{A}$ that winds up on $\mathrm{B}$, but rather an intervening water molecule acts as a catalyst: $\mathrm{AH}+\mathrm{OHH}+\mathrm{B} \rightarrow \mathrm{A}+\mathrm{HOH}+\mathrm{HB}$. To describe this process, one must obviously be able to make and break $\mathrm{OH}$ bonds, i.e., the $\mathrm{OH}$ bond degrees of freedom must be treated fully dynamically, and the quantum aspects of its dynamics will surely be significant.

Quantum dynamics of complex (large) molecular systems has thus been receiving a great deal of attention in the theoretical reaction dynamics community, and a variety of approaches are being developed and applications carried out. I will note some of these below as examples, but this is by no means a comprehensive review; I thus apologize beforehand for omissions that are certain to occur.

\section{Current Work in Quantum Dynamics of Complex Systems.}

First I note some fully rigorous quantum dynamics calculations that Makri and her coworkers ${ }^{16}$ have carried out for 'models' of complex systems, typically one (or a few) 'interesting' degree of freedom (e.g., the reaction coordinate for a chemical reaction) that is coupled to a 'bath' of harmonic oscillators that describe the environment (e.g., a cluster, a solid, a liquid, a protein framework, etc.). Makri et al. exploited the well-known procedure of integrating out the harmonic bath in a Feynman path integral formulation of the dynamics, and then more significantly they developed ways for doing the remaining path integral for the one 'interesting' degree of freedom (which is now far from trivial since it has memory effects due to its coupling to the bath). These calculations are of immense value in providing benchmark results for validating more approximate approaches (that can be applied to more realistic molecular systems); there are very few 'exact' quantum dynamics results for systems with many degrees of freedom to which one can compare approximate approaches. In some case, too, one can use a harmonic bath model to mimic real molecular systems, by choosing the distribution of frequencies and couplings to the harmonic modes to match those of the actual molecular environment; this has been done, for example, in a treatment of the photo-reaction center. ${ }^{17}$

Another exact quantum dynamics methodology that has made impressive contributions is the multi-configuration time-dependent Hartree (MCTDH) approach. Though the idea (of a multiconfiguration time-dependent self-consistent field approximation) was introduced and first applied earlier, ${ }^{18}$ it has been developed most effectively by the Heidelberg group. ${ }^{19}$ Though there are some limitations on the form of the Hamiltonian for which it is practical, it has been applied to molecular systems with tens of degrees of freedom and achieved excellent results. It, too, has provided benchmark results to which other more approximate approaches can be tested.

There are then a variety of approximate approaches that have been developed for dealing with complex systems, most of which can be described as 'mixed quantum-classical' methods, whereby one (or a few) 'interesting' or important degrees of freedom are treated quantum mechanically, e.g., by integrating the time-dependent Schrödinger equation for the wavefunction of these degrees of freedom, and the (many) remaining degrees of freedom treated by classical mechanics, i.e., by integrating classical equations of motion for their coordinates and momenta. The tricky part of these approaches is how one couples the quantum and classical degrees of 
freedom. There is much excellent work going on in this area, both in very sophisticated applications to bio-molecular systems, and also in new methodological developments. Some of the approaches are based on 'localized quantum transitions', and others utilize a variety of wavepacket (usually Gaussian) methods. Some of these approaches (e.g., various Gaussian wavepacket expansion methods) are formulated within an 'exact' framework, i.e., they would in principle lead to exact quantum results if the basis sets, etc., could be expanded without limit. The annual meeting of the American Chemical Society this past March in Atlanta had a weeklong symposium on quantum/classical calculations in chemistry and biophysics that contained many excellent papers in this area, and a recent workshop of CECAM (European Center for Atomic and Molecular Calculations) was on a similar topic, Development of Methods for Quantum Dynamics in the Condensed Phase. So there are a variety of things that can, and are, being done in this area..$^{20-41}$

Another approximate approach to quantum dynamics in complex systems is to use semiclassical (SC) theory to add quantum effects to classical MD simulations. This has the advantage of not having to divide the system into a quantum part and a classical part, since all degrees of freedom are treated equivalently. From the early SC work in the 1960's and 70's it seems clear that the SC approximation will provide a usefully accurate description of quantum effects in molecular dynamics; the outstanding challenge is to make them practical and efficient enough to be useful (the 'implementability' question). The initial value representation (IVR) of SC theory ${ }^{42-47}$ has re-emerged in this regard as the most promising way to accomplish this; it reduces the SC calculation to a phase space average over the initial conditions of classical trajectories, as it also required in a purely classical MD simulation. Thus one is able to leverage much of the Monte Carlo simulation technology that has been developed for classical MD in carrying out SC-IVR calculations. All of the beautiful (at least in some persons' eyes!), but complicated, analysis that has traditionally dominated SC theory-e.g., Airy function and other 'connection formulae', 'uniform' SC approximations, etc. - is 'swept under the rug' in the IVR approach, being subsumed in the Monte Carlo average over the phase space of initial conditions.

Numerous applications of the SC-IVR approach in recent years have established the fact that it does indeed provide a very useful description of quantum effects in molecular systems with many degrees of freedom. However, the calculations are more difficult to carry out than ordinary classical MD simulations, so that work is continuing to find more efficient ways to implement the SC-IVR. The next section describes some current work in this regard. I, and others, have published more comprehensive reviews of the SC-IVR and its applications, ${ }^{48-50}$ so the interested reader should consult these; the present paper is not such a review.

\section{SC-IVR Calculation of Time Correlation Functions.}

Most quantities of interest in the dynamics of complex systems can be expressed in terms of time correction functions ${ }^{51}$ of the form

$$
C_{A B}(t)=\operatorname{tr}\left[e^{-\beta \hat{H} / 2} \hat{A} e^{-\beta \hat{H} / 2} e^{i \hat{H} t / \hbar} \hat{B} e^{-i \hat{H} t / \hbar}\right]
$$

where $\hat{H}$ is the (time-independent) Hamiltonian of the complete molecular system, and $\hat{A}$ and $\hat{B}$ are operators relevant to the specific property of interest. [The Boltzmann operator does not 
need to be factored as it is in Eq. (1), but it is convenient in many cases to do so.] For example, if $\hat{A}=\hat{B}$ is the dipole moment operator, then the Fourier transform of the correlation function is the absorption spectrum; if it is the velocity operator of a tagged particle, or the flux operator related to a chemical reaction, then its time integral gives the diffusion coefficient and the chemical reaction rate, respectively,

The SC-IVR approximates the time evolution operator, exp $(-i \hat{H} t / \hbar)$, as follows

$$
e^{-i \hat{H} t / \hbar}=\int d \mathbf{p}_{0} \int d \mathbf{q}_{0} \sqrt{M_{q p} /(2 \pi i \hbar)^{F}} e^{i S_{t}\left(\mathbf{p}_{0}, \mathbf{q}_{0}\right) / \hbar}\left|\mathbf{q}_{t}><\mathbf{q}_{0}\right|
$$

where $\mathrm{F}$ is the number of degrees of freedom, $\left(\mathbf{p}_{0}, \mathbf{q}_{0}\right)$ are the initial coordinates and momenta for a classical trajectory, $\mathbf{q}_{\mathrm{t}}=\mathbf{q}_{\mathrm{t}}\left(\mathbf{p}_{0}, \mathbf{q}_{0}\right)$ is the coordinate (in the F-dimensional space) at time $\mathrm{t}$ which evolves from that trajectory, $S_{t}\left(\mathbf{p}_{0}, \mathbf{q}_{0}\right)$ is the classical action along the trajectory, and $M_{q p}$ is the determinant of the Jacobian matrix relating the final position and initial momentum,

$$
M_{q p}=\operatorname{det}\left[\partial \mathbf{q}_{t}\left(\mathbf{p}_{0}, \mathbf{q}_{0}\right) / \partial \mathbf{p}_{0}\right]
$$

(Eq. (2) is the original coordinate space, or Van Vleck IVR; a popular alternative is the coherent state, or Herman-Kluk IVR,$^{44}$ whereby the initial and final states are coherent states, and the preexponential Jacobian factor is also modified.) For the correlation function one needs to insert two such representation of the propagator into Eq. (1), yielding the following double phase space average for the correlation function,

$$
\begin{aligned}
C_{A B}(t)= & (2 \pi \hbar)^{-F} \int d \mathbf{p}_{0} \int d \mathbf{q}_{0} \int d \mathbf{p}_{0}{ }^{\prime} \int d \mathbf{q}_{0}{ }^{\prime}\left(M_{q p} M_{q p}{ }^{\prime}\right)^{1 / 2}<\mathbf{q}_{0}|\hat{A}(\beta)| \mathbf{q}_{0}{ }^{\prime}> \\
& e^{i S_{t}\left(\mathbf{p}_{0}, \mathbf{q}_{0}\right) / \hbar} e^{-i S_{t}\left(\mathbf{p}_{0}{ }^{\prime}, \mathbf{q}_{0}{ }^{\prime}\right) / \hbar}<\mathbf{q}_{t}{ }^{\prime}|\hat{B}| \mathbf{q}_{t}>.
\end{aligned}
$$

The primary difficulty in evaluating this expression is the oscillatory character of the integrand, coming from the difference of the action integrals from the trajectory with initial conditions $\left(\mathbf{p}_{0}, \mathbf{q}_{0}\right)$ and $\left(\mathbf{p}_{0}{ }^{\prime}, \mathbf{q}_{0}{ }^{\prime}\right)$.

\section{A. The Linearization Approximation.}

One way ${ }^{52}$ to deal with the problem of evaluating Eq. (3) is the (rather drastic) approximation of assuming that the dominant contribution to the double phase space average comes from phase points $\left(\mathbf{p}_{0}, \mathbf{q}_{0}\right)$ and $\left(\mathbf{p}_{0}{ }^{\prime}, \mathbf{q}_{0}{ }^{\prime}\right)$ - and thus the two trajectories emanating from them-that are close to one another. To effect this approximation one changes to the sum and difference variables

$$
\begin{aligned}
& \overline{\mathbf{p}}_{0}=\frac{1}{2}\left(\mathbf{p}_{0}+\mathbf{p}_{0}{ }^{\prime}\right) \\
& \overline{\mathbf{q}}_{0}=\frac{1}{2}\left(\mathbf{q}_{0}+\mathbf{q}_{0}{ }^{\prime}\right)
\end{aligned}
$$




$$
\begin{aligned}
& \Delta \mathbf{p}_{0}=\mathbf{p}_{0}-\mathbf{p}_{0}{ }^{\prime} \\
& \Delta \mathbf{q}_{0}=\mathbf{q}_{0}-\mathbf{q}_{0}{ }^{\prime}
\end{aligned}
$$

and then all quantities in the integrand of Eq. (3) are expanded to first order in $\Delta \mathbf{p}_{0}$ and $\Delta \mathbf{q}_{0}$; the integrals over $\Delta \mathbf{p}_{0}$ and $\Delta \mathbf{q}_{0}$ thus become Fourier integrals (since the phase of the integrand is linear in them), giving the linearized SC-IVR (LSC-IVR), or classical Wigner model for the correlation function,

$$
C_{A B}(t)=(2 \pi \hbar)^{-F} \int d \mathbf{p}_{0} \int d \mathbf{q}_{0} A_{w}^{\beta}\left(\mathbf{p}_{0}, \mathbf{q}_{0}\right) B_{w}\left(\mathbf{p}_{t}, \mathbf{q}_{t}\right)
$$

Here $\left(\mathbf{p}_{0}, \mathbf{q}_{0}\right)$ are the average values (i.e., the 'bars' have been removed), and $A_{w}$ and $B_{w}$ are the Wigner functions corresponding to these operators,

$$
O_{w}(\mathbf{p}, \mathbf{q}) \equiv \int d \Delta \mathbf{q} e^{i \mathbf{p} \cdot \Delta \mathbf{q} / \hbar}<\mathbf{q}-\Delta \mathbf{q} / 2|\hat{O}| \mathbf{q}+\Delta \mathbf{q} / 2>,
$$

for any operator $\hat{O}$.

Eq. (5) has the same form as the purely classical correlation function, the only difference being that the Wigner functions for operators $\hat{A}(\beta)$ and $\hat{B}$ appear rather than the corresponding classical functions. The classical Wigner model has been obtained many times before, by a variety of formulations. One such early paper is ref. 53, but it surely goes back further than this. Heller ${ }^{54}$ discussed the approximation many years ago (including an illuminating discussion of its limitations), and it was used by Lee and Scully ${ }^{55}$ to describe quantum effects in a collinear model of inelastic scattering. More recently it has been obtained form a different approach by Pollak, ${ }^{56}$ and also by Rossky et al. ${ }^{57}$ directly from a path integral representation of the two time evolution operators in Eq. (1) (again by linearizing in the difference between the two paths).

The importance of the above derivation is not the result itself, for as noted, the classical Wigner approximation has been around a long time, having been obtained from a variety of approaches. The important point is realizing that the classical Wigner model is contained within the SC-IVR description, and results from a very well defined approximation to it. And it also makes clear that if the SC-IVR can be implemented with less drastic approximations, it will be even more accurate than the classical Wigner model.

In many cases the operator $\hat{B}$ in the correlation function is a local operator (i.e., a function only of the coordinates of the system), and its Wigner function is simply the classical function itself. So the only difficulty in applying the LSC-IVR beyond a purely classical calculation is calculation of the Wigner function for operator $\hat{A}(\beta)$. This is non-trivial, however, since it involves a multi-dimensional Fourier transform involving the Boltzmann operator, exp $(-\beta \hat{H})$, of the complete system. In some early applications ${ }^{52 b}$ the Boltzmann operator was approximated as harmonic (about the saddle point on a potential energy surface), and this is fine so long as the temperature is not too low. $\mathrm{Geva}^{58}$ has developed a less drastic approximation that involves only 
a local (rather than global) harmonic approximation, and Rossky et al. ${ }^{57}$ have used the FeynmanKleinert approach of making a variational harmonic approximation. And Coker et al. ${ }^{59}$ have extended this latter approach also to be able to describe electronically non-adiabatic dynamics. All these types of harmonic approximations allow the Fourier transform to be evaluated analytically, so the Wigner function $\hat{A}_{w}(\beta)$ is readily obtained, and the LSC-IVR calculation then becomes essentially the same level of difficulty as a classical one.

As drastic as this linearization approximation seems, it is surprising how well it can work. E.g., Figure 1 shows how it describes tunneling for a standard 1d model problem, a potential barrier with parameters and particle mass corresponding approximately to the $\mathrm{H}+\mathrm{H}_{2}$ reaction. $^{60}$ The Arrhenius plot of the rate shows the expected good agreement with the exact quantum rate at higher temperature when tunneling corrections are small, but even at lower temperature where tunneling corrections become significant it does very well: at $300 \mathrm{~K}$, where the tunneling correction factor is $\sim 2.5$, the rate given by the LSC-IVR is only $10 \%$ too small, and at the lowest temperature shown $(200 \mathrm{~K})$, where the tunneling correction is a factor of $\sim 2000$, it is only $35 \%$ too small. (The full SC-IVR calculation is accurate to a few $\%$ even down to $200 \mathrm{~K}$.)

Figure 2 shows the rate constant for isomerization in a double well potential that is coupled to a bath of harmonic oscillators ${ }^{52 \mathrm{~b}}$ (with the Boltzmann operator being approximated as harmonic about the transition state in order to compute the Wigner function of operator $\hat{A}(\beta), \hat{A}$ being the flux operator in this case). The rate is shown as a function of the coupling between the reaction coordinate and the bath (the 'friction'), and excellent agreement is shown with Makri et al.' $\mathrm{s}^{61}$ exact benchmark results in both the low friction, Lindemann 'fall-off' regime, and also in the high friction 'Kramer's turn-over' regime. And as noted above, Geva et al. ${ }^{58}$ have developed more accurate ways of evaluating the Fourier transform to obtain the Wigner function involving the Boltzmann operator and carried out some impressive applications for calculating vibrational relaxation in liquids (where the relevant quantity is a force-force correlation function). And Rossky et al. ${ }^{57}$ (using the variational harmonic approximation to obtain the Wigner function involving the Boltzmann operator) have treated a 1-dimensional chain of helium atoms, and also liquid oxygen $\left(32 \mathrm{O}_{2}\right.$ molecules in a box) at low temperature $(70 \mathrm{~K})$.

It should also be noted that there are several other approaches that are very similar in character to the LSC-IVR/classical Wigner model, though not identical to it. E.g., the 'forwardbackward' approximation to the SC-IVR correlation function used very effectively by Makri et $a{ }^{62}$ is closely related to it, and the centroid molecular dynamics approach developed by Voth $e t$ $a l .{ }^{63}$ has very similar behavior. All of these approaches share the ability of the LSC-IVR to describe some of the quantum mechanical aspects of molecular dynamics, but they are not able to describe quantum coherence effects. Coherence effects arise (in a semiclassical picture) from the interference between different trajectories; and since the LSC-IVR only considers trajectories in the double phase space average [Eq. (3)] that are infinitesimally close to one another, such coherence effects are explicitly excluded within this approximation.

\section{B. A Forward-Backward SC-IVR.}

An approximation that is intermediate between the full SC-IVR correlation function of Eq. (3) and its linearized approximation of Eq. (5) - both in accuracy and in difficulty - is the 
forward-backward IVR as developed by Miller et al. ${ }^{64}$ The idea came from some earlier work of Makri et al. ${ }^{65}$ but was implemented in a different way that is more accurate (and also more difficult to apply), and most importantly is able to describe coherence effects. One follows somewhat the same goal as in the linearized approximation, i.e., one wishes to cancel out the unnecessary oscillations between the forward trajectory, that propagates from 0 to $t$, and the backward trajectory that propagates from $t$ back to 0 , but without making the drastic approximation that the forward and backward trajectories are infinitesimally close to one another.

This FB-IVR approach assumes that operator $\hat{B}$ in the correlation function involves only a few (perhaps collective) degrees of freedom, as is often the case. For example, if $B(\mathbf{q})$ is a function of the form

$$
B(\mathbf{q})=B(s(\mathbf{q})),
$$

then the FB-IVR result for the correlation function is ${ }^{64}$

$$
C_{A B}(t)=\int_{-\infty}^{\infty} d p_{s} \tilde{B}\left(p_{s}\right)(2 \pi \hbar)^{-F} \int d \mathbf{p}_{0} \int d \mathbf{q}_{0} C\left(\mathbf{p}_{0}, \mathbf{q}_{0} ; p_{s}\right)<\mathbf{p}_{0}, \mathbf{q}_{0}|\hat{A}(\beta)| \mathbf{p}_{0}{ }^{\prime}, \mathbf{q}_{0}{ }^{\prime}>e^{i S\left(\mathbf{p}_{0}, \mathbf{q}_{0} ; p_{s}\right) / \hbar},
$$

where here it is more convenient to use the coherent state (Herman-Kluk) IVR. ( $\left.\mathbf{p}_{0}, \mathbf{q}_{0}\right)$ in Eq. (8) are the initial conditions for a trajectory that is evolved to time $t$ in the usual way, but here the momentum vector undergoes a momentum jump according as follows

$$
\mathbf{p}_{t} \rightarrow \mathbf{p}_{t}+\frac{\partial s\left(\mathbf{q}_{t}\right)}{\partial \mathbf{q}_{t}} p_{s}
$$

and the trajectory is then propagated back to time $0 ;\left(\mathbf{p}_{0}^{\prime}, \mathbf{q}_{0}^{\prime}\right)$ is the final phase point of this forward-backward trajectory, $\mathrm{S}$ the classical action along it, and $\mathrm{C}$ the Herman-Kluk preexponential factor; $\tilde{B}\left(p_{s}\right)$ is the (1-dimensional) Fourier transform of $\mathrm{B}(\mathrm{s})$.

This FB-IVR result involves only a 1-parameter integral in addition to a (single) phase space average over initial conditions, and is thus perhaps the simplest result of all that is capable of describing quantum coherence. To see that it can, consider Figures 3 and 4 . This is the result of a FB-IVR calculation ${ }^{66}$ for a time-dependent radial distribution function, i.e., a model for femtosecond structure determination. The specific model is a Morse potential (with parameters corresponding to the B-state of $\mathrm{I}_{2}$ ) coupled to a harmonic bath (modeling the environmental degrees of freedom, e.g. a cluster, a liquid, etc.) $\mid \phi>$ is the ground vibrational state of the diatomic in the ground electronic state, which becomes (upon Franck-Condon excitation) the initial vibrational wavefunction in the B-state. The time-dependent radial distribution function-i.e., the probability distribution of the diatomic coordinate at time $\mathrm{t}-$ is given by the correlation function of Eq. (1), where operators $\hat{A}(\beta)$ and $\hat{B}$ are

$$
\hat{A}(\beta)=|\phi><\phi| e^{-\beta \hat{H}_{b}}
$$




$$
\hat{B}(r)=\delta(r-\hat{r})
$$

where $\hat{H}_{b}$ is the Boltzmann operator for the harmonic bath. The correlation $\mathrm{C}_{\mathrm{AB}}(\mathrm{t})$ is then $\mathrm{P}_{\mathrm{t}}(\mathrm{r})$, the probability distribution of the diatomic coordinate at time $t$, i.e., the radial distribution function of $\mathrm{I}_{2}$. Fig. 3 shows this for a time of $192 \mathrm{fsec}$ (about $1 \frac{1}{4}$ vibrational periods of $\mathrm{I}_{2}$ ) for the isolated diatomic (i.e., no coupling to the bath), and one sees very pronounced coherence structure (due to the fact that the initial state is a coherent superposition of many different vibrational eigenstates of the B-state); the FB-IVR result is essentially indistinguishable from that of the exact quantum calculation (which is easy for the isolated diatom case). Also shown is the result of the LSC-IVR/classical Wigner calculation, which shows none of the coherence structure. Fig. 4 then shows $\mathrm{P}_{\mathrm{t}}(\mathrm{r})$ with coupling to the bath, for several values of the bath temperature $\mathrm{T}$. For $\mathrm{T}=0$, the result is essentially the same as the isolated molecule result of Fig. 3 , i.e., the bath is 'frozen out'. But as $\mathrm{T}$ is increased, the coherence structure is progressively quenched (or 'de-coherred') by coupling to the bath, and by the time it has increased to $300 \mathrm{~K}$ the coherence features have mostly disappeared (for the assumed coupling strength), and in this case one sees that the LSC-IVR does an excellent job in describing $\mathrm{P}_{t}(\mathrm{r})$. So just as one would expect, when quantum coherence features are averaged out, classical mechanics (which is effectively what the LSC-IVR gives) works well. This is not a surprising result. The point of this example is to show that semiclassical theory is able to simulate these coherence effects (and the extent to which they are quenched) in systems with many degrees of freedom. This model system is of course a simple one, but the nature of the calculation for a realistic model of a large molecular system would be essentially the same (though the computational time for each trajectory would of course be greater for a more complicated potential energy surface).

\section{An Exact Forward-Backward IVR.}

Finally, it is interesting to show that there is an exact forward-backward semiclassical IVR expression (EFB-IVR) for the time correlation function, and this in many ways it is not more complex than the linearized approximation (LSC-IVR) to it. For simplicity here I will use notation for a single coordinate and momentum, but the generalization to many degrees of freedom is obvious (and essentially identical). Also, operator $\hat{B}$ will be assumed to be local, i.e., $\hat{B}=\mathrm{B}(\mathrm{q})$, but this can be generalized if needed.

Writing out the coordinate representation of Eq. (1) explicitly gives

$$
C_{A B}(t)=\int d q_{0} \int d q_{0}{ }^{\prime} \int d q<q_{0}|\hat{A}(\beta)| q_{0}{ }^{\prime}><q_{0}{ }^{\prime}\left|e^{i \hat{H} t / \hbar}\right| q>B(q)<q\left|e^{-i \hat{H} t / \hbar}\right| q_{0}>,
$$

and the standard (Van Vleck) SC approximation for the time evolution operators is

$$
\begin{aligned}
& <q\left|e^{-i \hat{H} t / \hbar}\right| q_{0}>=\sum\left(2 \pi i \hbar M_{q p}\right)^{-1 / 2} e^{i S_{t}\left(q, q_{0}\right) / \hbar} \\
& <q_{0}{ }^{\prime}\left|e^{i \hat{H} t / \hbar}\right| q>=\sum\left(2 \pi i \hbar M_{q p}{ }^{\prime}\right)^{-1 / 2} e^{i S_{-t}\left(q_{0}^{\prime}, q\right) / \hbar}
\end{aligned}
$$

where the sums are over all trajectories that go from $\mathrm{q}_{0}$ to $\mathrm{q}$ in time $\mathrm{t}$, and from $\mathrm{q}$ to $\mathrm{q}_{0}$ ' in time 
-t, respectively. One now uses the IVR 'trick' 49 twice in succession; first, the integral over $q$ (and the sum over multiple trajectories in the forward time propagator) is replaced by an integral over the initial momentum $\mathrm{p}_{0}$,

$$
\sum \int d q \rightarrow \int d p_{0}\left|M_{q p}\right|
$$

coordinate $\mathrm{q} \equiv \mathrm{q}_{\mathrm{t}}\left(\mathrm{p}_{0}, \mathrm{q}_{0}\right)$ is the position reached by the trajectory with initial conditions $\left(\mathrm{p}_{0}, \mathrm{q}_{0}\right)$. At time $t$ one then replaces the integral over $\mathrm{q}_{0}{ }^{\prime}$ (and the sum over multiple trajectories in the backward propagator) by one over $\mathrm{p}_{\mathrm{t}}^{\prime}$,

$$
\sum \int d q_{0}{ }^{\prime} \rightarrow \int d p_{t}{ }^{\prime}\left|M_{q p}{ }^{\prime}\right|
$$

so that the final expression for the correlation function is

$$
C_{A B}(t)=(2 \pi \hbar)^{-1} \int d p_{0} \int d q_{0} \int d p_{t}{ }^{\prime}\left(-M_{q p} M_{q p}{ }^{\prime}\right)^{1 / 2}<q_{0}|\hat{A}(\beta)| q_{0}{ }^{\prime}>e^{i S_{-t}\left(p_{t}{ }^{\prime}, q_{t}\right) / \hbar} e^{i S_{t}\left(p_{0}, q_{0}\right) / \hbar} B\left(q_{t}\right)
$$

The structure of Eq. (15) is as follows: $\left(\mathrm{p}_{0}, \mathrm{q}_{0}\right)$ are the initial conditions for a trajectory that is propagated forward to time $t$, arriving at $\mathrm{q}_{\mathrm{t}}\left(\mathrm{p}_{0}, \mathrm{q}_{0}\right) ; \mathrm{S}_{\mathrm{t}}\left(\mathrm{p}_{0}, \mathrm{q}_{0}\right)$ is the action integral along it, and $\mathrm{M}_{\mathrm{qp}}$ $=\partial \mathrm{q}_{\mathrm{t}}\left(\mathrm{p}_{0}, \mathrm{q}_{0}\right) / \partial \mathrm{p}_{0}$ the usual Jacobian factor. At time $\mathrm{t}$ the momentum (which has the value $\left.\mathrm{p}_{\mathrm{t}}\left(\mathrm{p}_{0}, \mathrm{q}_{0}\right)\right)$ is replaced by $\mathrm{p}_{\mathrm{t}}{ }^{\prime}$ and the trajectory then propagated backward in time for $-\mathrm{t}$ (i.e., the final time is again 0$)$, arriving at position $\mathrm{q}_{0}{ }^{\prime}\left(\mathrm{p}_{\mathrm{t}}{ }^{\prime}, \mathrm{q}_{\mathrm{t}}\right) ; \mathrm{S}_{\mathrm{t}}\left(\mathrm{p}_{\mathrm{t}}{ }^{\prime}, \mathrm{q}_{\mathrm{t}}\right)$ is the action integral along this backward trajectory, and $\mathrm{M}_{\mathrm{qp}}{ }^{\prime}=\partial \mathrm{q}_{0}{ }^{\prime}\left(\mathrm{p}_{\mathrm{t}}{ }^{\prime}, \mathrm{q}_{\mathrm{t}}\right) / \partial \mathrm{p}_{\mathrm{t}}{ }^{\prime}$ the usual Jacobian. The variables $\mathrm{p}_{0}, \mathrm{q}_{0}, \mathrm{p}_{\mathrm{t}}{ }^{\prime}$ are integrated over. (Fig. 5 shows this forward-backward structure pictorially.)

It is important to emphasize that the EFB-IVR, Eq. (15), is completely equivalent to Eq. (11), i.e., it entails no approximation other than the basis SC (Van Vleck) approximation itself. It is also interesting to note the similarity of it to the corresponding linearized approximation (the LSC-IVR), Eq. (5), which is as follows if written out explicitly for the present 1d case,

$$
C_{A B}(t)=(2 \pi \hbar)^{-1} \int d p_{0} \int d q_{0} \int d \Delta q<q_{0}-\frac{\Delta q}{2}|\hat{A}(\beta)| q_{0}+\frac{\Delta q}{2}>e^{i p_{0} \Delta q / \hbar} B\left(q_{t}\right)
$$

Note that both the EFB-IVR (Eq. (15)) and the LSC-IVR (Eq. (16)) involve three integration variables, and the integrand of both contains a phase factor (that is the most troublesome part of the calculation). Furthermore, it is easy to see how the EFB-IVR reduces to the linearized approximation by assuming that $p_{t}^{\prime}$ is close to $p_{t}$, i.e., that the backward trajectory is infinitesimally close to the forward one; specifically, if one changes the integration variable $p_{t}^{\prime}$ to $\Delta \mathrm{p}$,

$$
p_{t}{ }^{\prime}=p_{t}\left(p_{0}, q_{0}\right)+\Delta p
$$

and linearizes the phase (and other variables) in $\Delta \mathrm{p}$; the LSC-IVR then results.

The essential complexity of the EFB-IVR calculation is thus no greater than that of the LSCIVR, so that the methods that have been developed for dealing with the LSC-IVR approximation 
should also be useful in dealing with the more rigorous EFB-IVR version of the theory. Work in progress ${ }^{67}$ using the modified Filinov filtering technique is actually able to 'tune' the expression for the correlation function continuously between its 'exact' form (the EFB-IVR, Eq. (15)) and the linearization approximation to it (Eq. (16)).

\section{Concluding Remarks}

There are certainly many phenomena for which the quantum mechanical aspects of molecular dynamics are unimportant, but one can never know this unless one has the ability to include quantum effects in the dynamics, even if only approximately. And there are also certain situations for which one knows quantum effects will be important. Much effort is thus being devoted to developing approximate ways for carrying out quantum dynamics simulations; this overview has focused primarily of one class of such approximations, namely the semiclassical approximation as implemented via an initial value representation.

A number of simple systems, and models of complex systems (i.e., a reaction coordinate coupled to many harmonic degrees of freedom) have been treated via SC-IVR's and compared to accurate results for these problems, demonstrating the feasibility of applying the approach to systems with many degrees of freedom and showing that it provides a very useful description of quantum effects in such systems. Furthermore, several groups have carried out impressive calculations using the simplest version of the theory, the linearized approximation (LSCIVR)/classical Wigner model, for 'real' (i.e., realistic models of) molecular systems. In many cases this classical Wigner model is adequate, but there are some quantum phenomena that it is not capable of describing (specifically, quantum coherence effects). It was shown that there exists an 'exact' (in the sense of no approximation beyond the SC approximation itself) forwardbackward version of the SC-IVR for time correlation functions that is very similar in structure to the simpler (approximate) LSC-IVR. This EFB-IVR is capable of describing all quantum effects in molecular dynamics, so attention is now being focused on using the developments that have made the LSC-IVR practical also to implement the EFB-IVR.

\section{Acknowledgments}

This work was supported by the Director, Office of Science, Office of Basic Energy Sciences, Chemical Sciences, Geosciences, and Biosciences Division, U.S. Department of Energy under Contract No. DE-AC03-76SF00098 and by the National Science Foundation Grant No. CHE-0345280.

\section{References}

1. See Adv. Chem. Phys. 10 (1966) for an excellent survey of the early history of crossed molecular beam research in chemistry.

2. E. H. Taylor and S. Datz, J. Chem. Phys. 23, 1711 (1955).

3. E. F. Greene, R. W. Roberts, and J. Ross, J. Chem. Phys. 32, 940 (1960).

4. D. R. Herschbach, G. H. Kwei, and J. A. Norris, J. Chem. Phys. 34, 1842 (1961).

5. A. E. Grosser, A. R. Blythe, and R. B. Bernstein, J. Chem. Phys. 42, 1268 (1964). 
6. For example, D. M. Neumark, A. M. Wodtke, G. N. Robinson, C. C. Hayden, K. Shobatake, R. K. Sparks, T. D. Schaefer, and Y. T. Lee, J. Chem. Phys. 82, 3067 (1985).

7. G. C. Schatz, this issue.

8. B. J. Alder and T. E. Wainwright, J. Chem. Phys. 31, 459 (1959).

9. (a) N. C. Balis and D. L. Bunker, J. Chem. Phys. 39, 315 (1963).

(b) M. Karplus, R. N. Porter, and R. D. Sharma, J. Chem. Phys. 40, 2033 (1964).

10. B. J. Alder, private communication.

11. K. W. Ford and J. A. Wheeler, Ann. Phys. (N.Y.) 7, 259, 287 (1959).

12. R. B. Bernstein, Adv. Chem. Phys. 10, 75 (1966).

13. See for example, P. E. Siska, J. M. Parson, T. P. Schaefer, and Y. T. Lee, J. Chem. Phys. 55, 5762 (1971).

14. W. H. Miller, (a) J. Chem. Phys. 53, 1949, 3578 (1970); (b) For a review, see Adv. Chem. Phys. 25, 69 (1974).

15. R. A. Marcus, (a) Chem. Phys. Lett. 7, 525 (1970); (b) J. Chem. Phys. 54, 3965 (1971).

16. N. Makri, J. Phys. Chem. A 102, 4414 (1998).

17. E. Sim and N. Makri, J. Phys. Chem. B 101, 5446 (1997).

18. N. Makri and W. H. Miller, J. Chem. Phys. 87, 5781 (1987).

19. (a) H. D. Meyer and G. A. Worth, Theor. Chem. Acc. 109, 251 (2003).

(b) M. H. Beck, A. Jackle, G. A. Worth, and H. D. Meyer, Phys. Rep. 324, 1 (2000).

20. M. Ben-Nun, J. Quenneville, and T. J. Martinez, J. Phys. Chem. A 104, 5161 (2000).

21. S. Y. Kim and S. J. Hammes-Schiffer, J. Chem. Phys. 119, 4389 (2003).

22. H. Wang and M. Thoss, J. Phys. Chem. A 107, 2126 (2003).

23. P. H. Nguyen and G. Stock, J. Chem. Phys. 119, 11350 (2003).

24. Y. Wu and V. S. Batista, J. Chem. Phys. 121, 1676 (2004).

25. D. V. Shalashilin, M. S. Child and D. C. Clary, J. Chem. Phys. 120, 5608 (2004).

26. Q. Shi and E. Geva, J. Chem. Phys. 121, 3393 (2004).

27. N. J. Wright and N. Makri, J. Chem. Phys. 119, 1634 (2003).

28. A. Donoso and C. C. Martens, J. Chem. Phys. 116, 10598 (2002).

29. O. V. Prezhdo and Y. V. Pereverzev, J. Chem. Phys. 113, 6557 (2000).

30. T. D. Hone and G. A. Voth, J. Chem. Phys. 121, 6412 (2004).

31. S. Bonella and D. F. Coker, J. Chem. Phys. 114, 7778 (2001).

32. A. E. Cardenas, R. Krems, and R. D. Coalson, J. Phys. Chem. A 103, 9469 (1999).

33. L. Turi and P. J. Rossky, J. Chem. Phys. 120, 3688 (2004).

34. M. Nest and P. Saalfrank, Chem. Phys. 268, 65 (2001).

35. S. Jan and J. Cao, J. Chem. Phys. 114, 9959 (2001).

36. A. Sergi, D. M. Kernan, G. Ciccotti, and R. Kapral, Theor. Chem. Acc. 110, 49 (2003).

37. D. E. Makarov and H. Metiu, J. Chem. Phys. 111, 10126 (1999).

38. J. Zhang and E. Pollak, J. Chem. Phys. 119, 11058 (2003).

39. D. Antoniou and S. D. Schwartz, J. Chem. Phys. 110, 7359 (1999).

40. J. Gao, and D. G. Truhlar, Ann. Rev. Phys. Chem. 53, 467 (2002).

41. M. H. M. Olsson, P. E. M. Siegbahn, and A. Warshel, J. Am. Chem. Soc. 126, 2820 (2004).

42. I. R. Craig and D. E. Manolopoulos, J. Chem. Phys. 121, 3368 (2004).

43. W. H. Miller, (a) J. Chem. Phys. 53, 3578 (1970); (b) J. Chem. Phys. 95, 9428 (1991).

44. M. F. Herman and E. Kluk, Chem. Phys. 91, 27 (1984).

45. E. J. Heller, (a) J. Chem. Phys. 94, 2723 (1991); (b) J. Chem. Phys. 95, 9431 (1991).

46. K. G. Kay, (a) J. Chem. Phys. 100, 4377 (1994); (a) J. Chem. Phys. 100, 4432 (1994). 
47. G. Capolieti and P. Brumer, Phys. Rev. A 50, 997 (1994).

48. D. J. Tannor and S. Garaschuk, Ann. Rev. Phys. Chem. 51, 553 (2000).

49. W. H. Miller, (a) J. Phys. Chem. A 105, 2942 (2001); (b) Proc. Natl. Acad. Sci. 102, 6660 (2005).

50. K. G. Kay, Ann Rev. Phys. Chem. 56, 255 (2005).

51. B. J. Berne and C. D. Harp, Adv. Chem. Phys. 17, 63 (1970).

52. (a) X. Sun and W. H. Miller, J. Chem. Phys. 106, 916 (1997).

(b) H. B. Wang, X. Sun, and W. H. Miller, J. Chem. Phys. 108, 9726 (1998).

53. K. Imne, E. Ozizmir, M. Rosenbaum, and P. F. Zweifel, J. Math. Phys. 8, 10907 (1967).

54. (a) E. J. Heller, J. Chem. Phys. 65, 1289 (1976).

(b) E. J. Heller and R. C. Brown, J. Chem. Phys. 75, 1048 (1976).

55. H. W. Lee and M. O. Scully, J. Chem. Phys. 73, 2238 (1980).

56. J. L. Liao and E. Pollak, J. Chem. Phys. 108, 2733 (1998).

57. J. A. Poulsen, G. Nyman, and P. J. Rossky, J. Chem. Phys. 119, 12179 (2003).

58. Q. Shi and E. Geva, J. Chem. Phys. 120, 10647 (2004).

59. S. Bonella and D. F. Coker, Proc. Natl. Acad. Sci. 102, 6715 (2005).

60. T. Yamamoto, H. B. Wang, and W. H. Miller, J. Chem. Phys. 116, 7335 (2002).

61. M. Topaler and N. Makri, J. Chem. Phys. 101, 7500 (1994).

62. N. Makri, A. Nakayama, and N. J. Wright, J. Theo. Comp. Chem. 3, 391 (2004).

63. J. Cao and G. A. Voth, J. Chem. Phys. 99, 10070 (1993).

64. W. H. Miller, Faraday Disc. Chem. Soc. 110, 1 (1998).

65. N. Makri and K. Thompson, Chem. Phys. Lett. 291, 101 (1998).

66. H. Wang, M. Thoss, K. Sorge, R. Gelabert, X. Gimenez, and W. H. Miller, J. Chem. Phys. 114, 2562 (2001).

67. C. Venkataraman and W. H. Miller, work in progress.

\section{Figure Captions}

Figure 1. Arrhenius plot of the rate constant for a 1d Eckart barrier (with parameters correspondingly approximately to the $\mathrm{H}+\mathrm{H}_{2}$ reaction). The solid line is the exact quantum value and the dashed line that given by classical mechanics. The circles are values given by the full SC-IVR and the squares its linearized approximation (LSC-IVR).

Figure 2. Transmission coefficient, $\kappa \equiv \mathrm{k} / \mathrm{k}_{0}$, for the isomerization rate in a double well potential coupled to a harmonic bath ( $\mathrm{k}$ is the rate constant, and $k_{0}=\frac{\omega_{0}}{2 \pi} e^{-V_{0} / k T}$ the $1 \mathrm{~d}$ classical rate), as a function of coupling to the bath. The solid lines are the results of the linearized approximation to the SC-IVR (LSC-IVR), and the solid points the accurate quantum values of Topaler and Makri, ref. 61 . (a) $\mathrm{T}=300 \mathrm{~K}$ (b) $\mathrm{T}=200 \mathrm{~K}$.

Figure 3. Probability distribution of the vibrational coordinate of $\mathrm{I}_{2}$ (modeled as a $1 \mathrm{~d}$ Morse oscillator) at time $\mathrm{t}=192 \mathrm{fsec}\left(\sim 1 \frac{1}{4}\right.$ vibrational periods after excitation). The dashed line and solid line (almost indistinguishable) are the exact quantum and forward-backward SC-IVR (FBIVR) results, respectively, and the dash-dot line the results of linearized approximation to the SC-IVR (LSC-IVR). 
Figure 4. Same quantity as Fig. 3, but with the addition of a harmonic bath that is coupled to the Morse oscillator $\left(\mathrm{I}_{2}\right)$; T is the temperature of the harmonic bath. The solid line is the result of the FB-IVR, and the dash-dot line that of the LSC-IVR.

Figure 5. Schematic depiction of the forward and backward trajectories in the 'exact' forwardbackward (EFB-IVR) approach described in Section III C. The trajectory begins at $t=0$ with initial conditions $\left(\mathrm{p}_{0}, \mathrm{q}_{0}\right)$ and is evolved to time $\mathrm{t}$, where its coordinates and momenta are $\left(\mathrm{p}_{\mathrm{t}}, \mathrm{q}_{\mathrm{t}}\right)$; here the momentum is changed to $p_{t}^{\prime}$ and the trajectory evolved backward in time to $t=0$, where its values are $\left(\mathrm{p}_{0}{ }^{\prime}, \mathrm{q}_{0}{ }^{\prime}\right)$. 


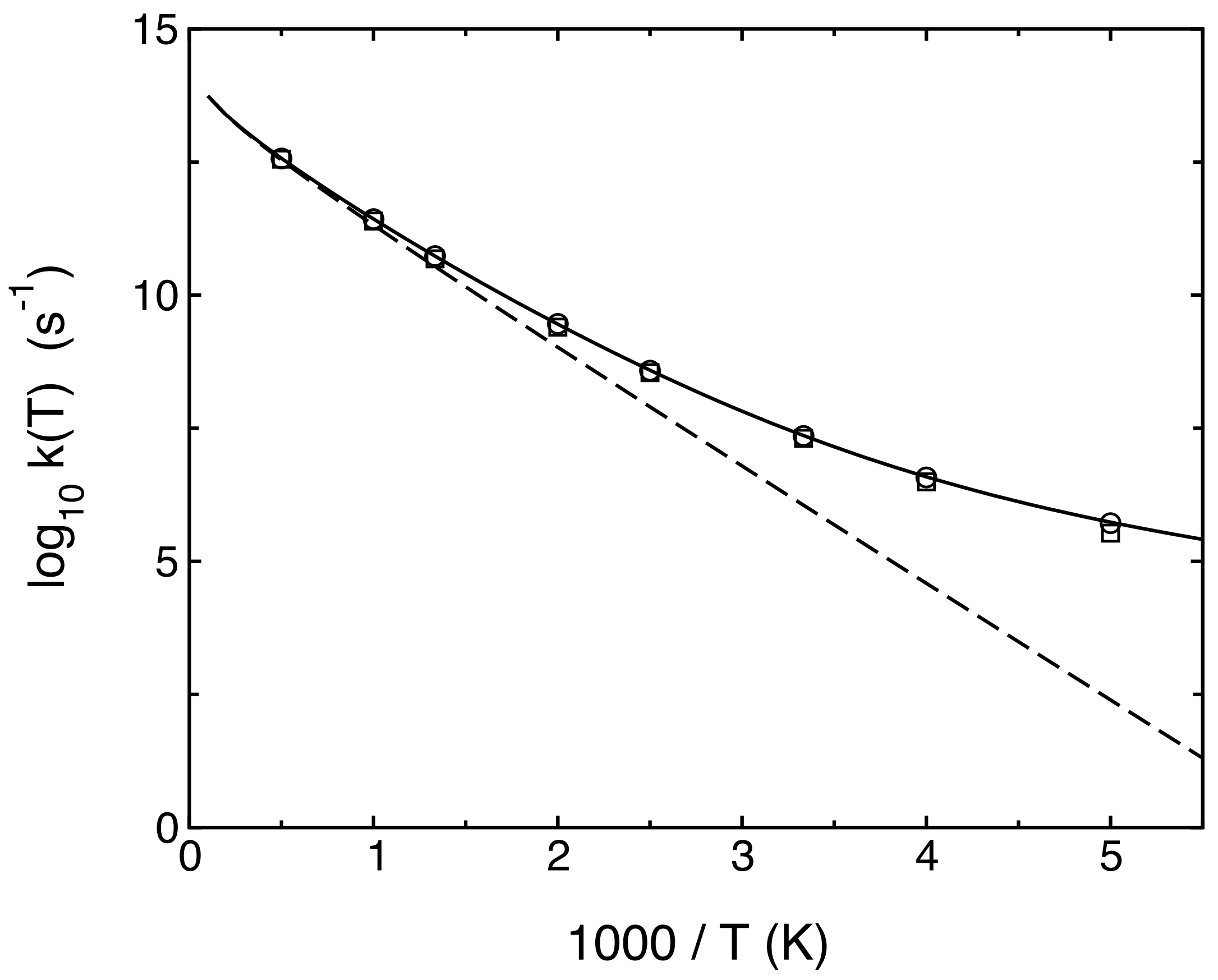



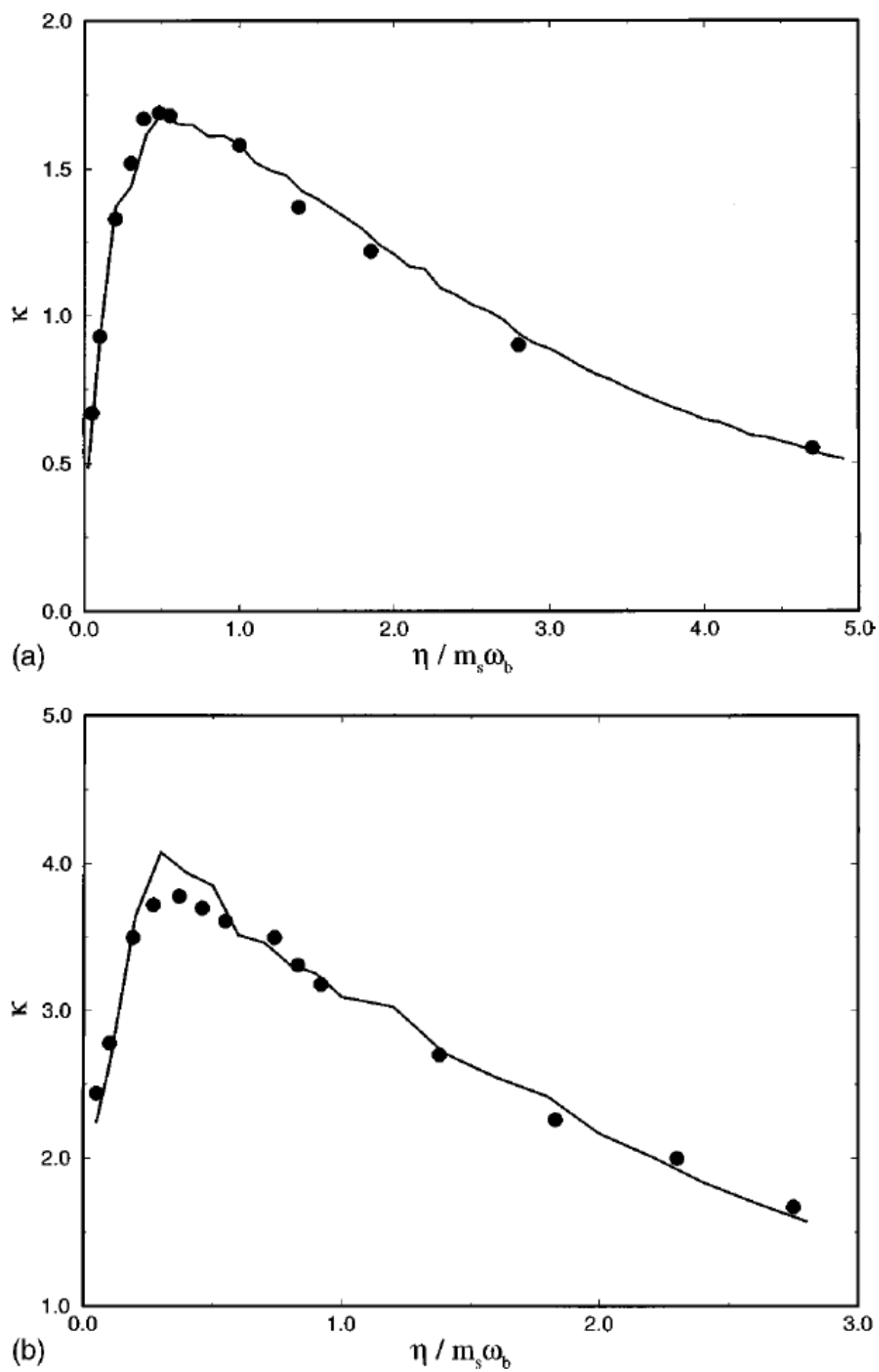


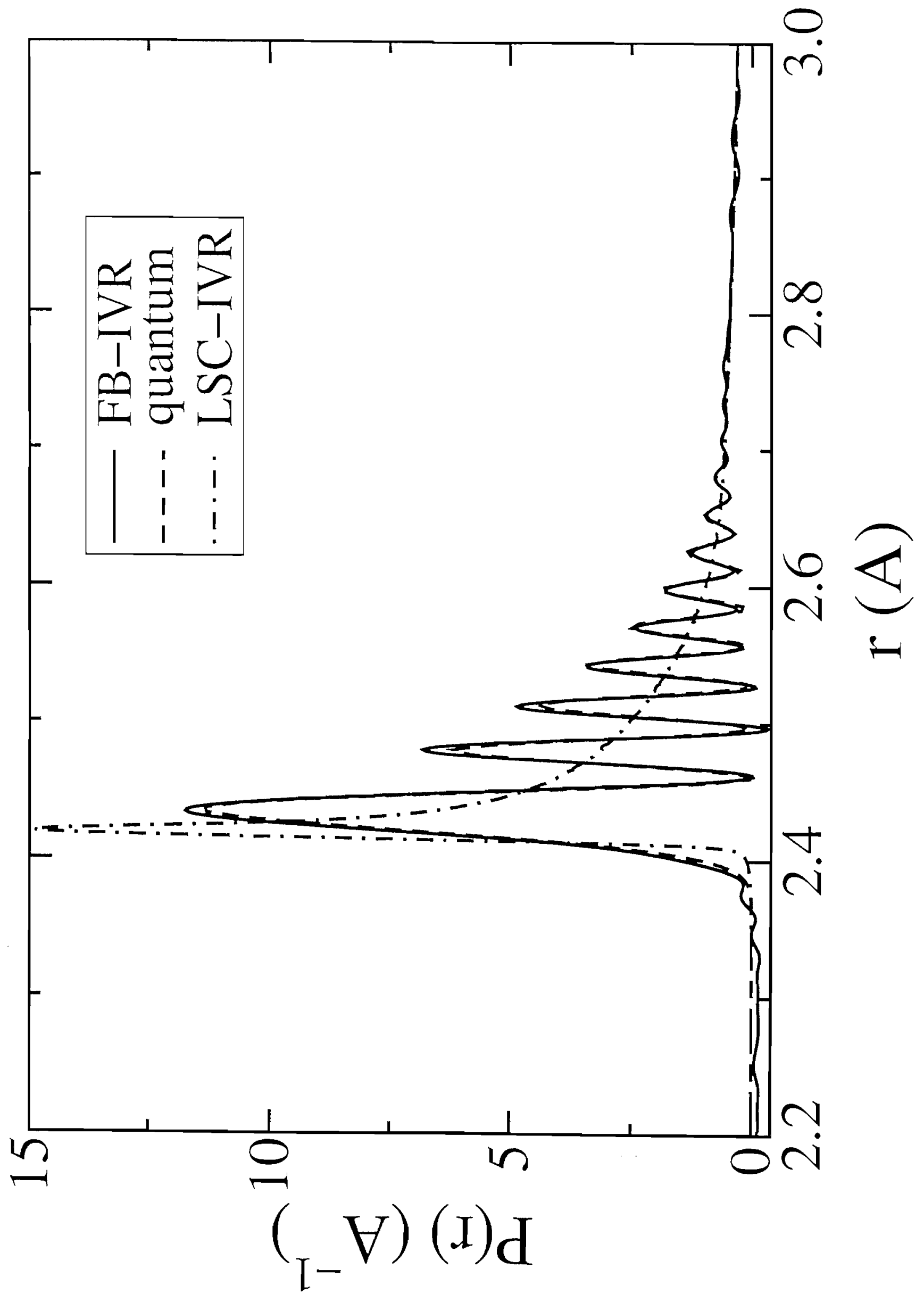



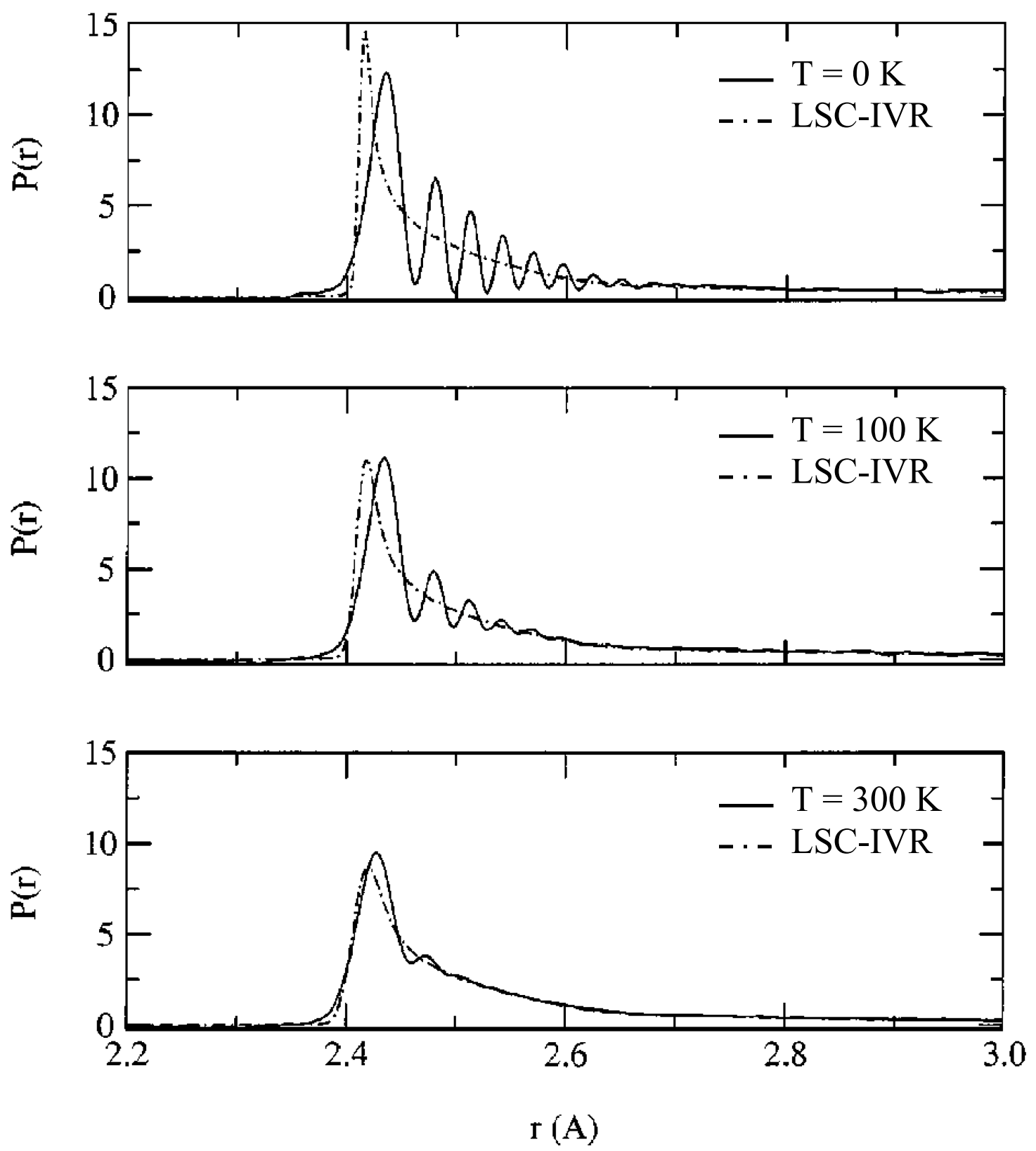


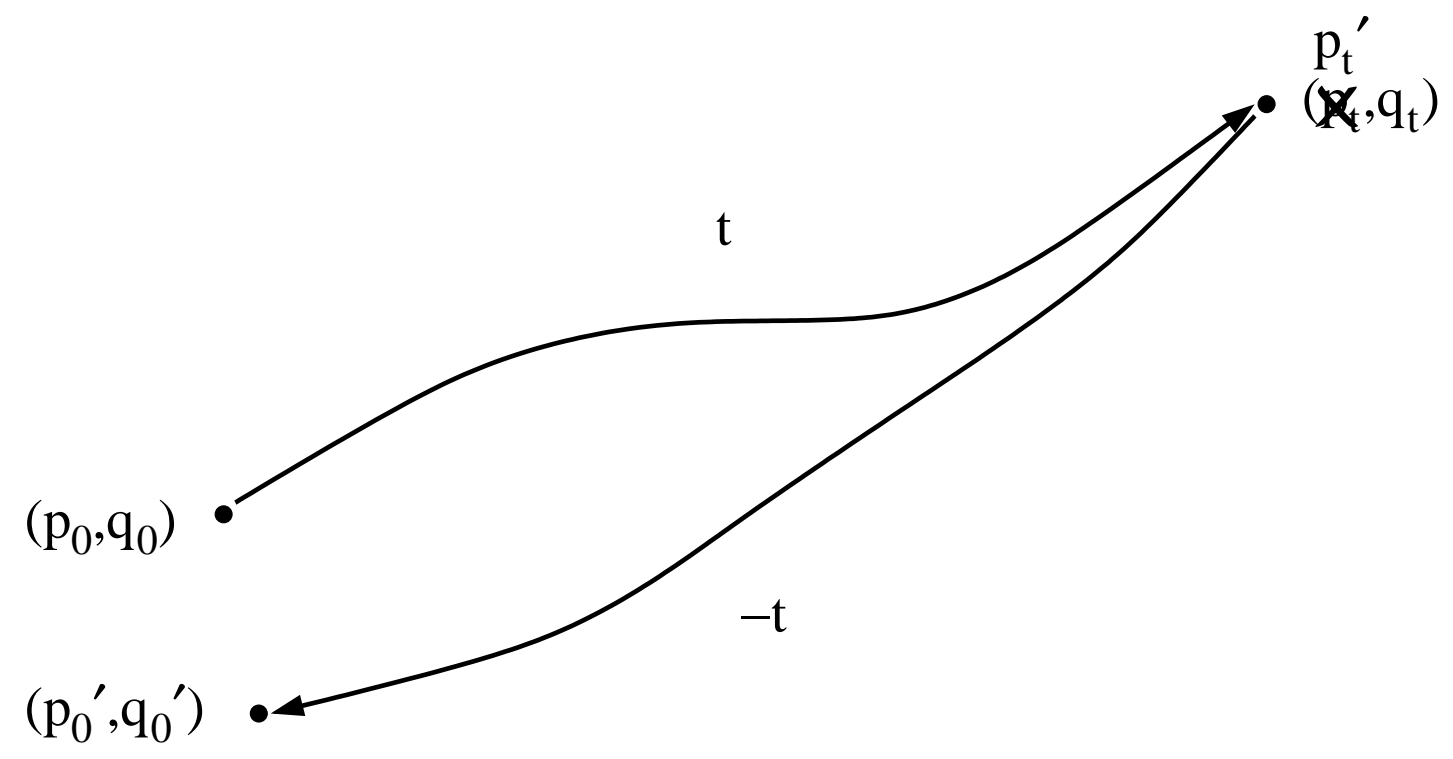

